\title{
THE RESISTANCE OF AN ELECTRICAL NETWORK
}

\section{R. M. $\mathrm{COHN}$}

1. Introduction. We consider a two-terminal electrical network ${ }^{1} N$ of branches $w_{1}, w_{2}, \cdots, w_{n}$, having the independently variable resistances $r_{1}, r_{2}, \cdots, r_{n}$ respectively. The resistance $R$ measured between the terminals of this network is a homogeneous rational function of the $r_{i}$. We shall show that $R$ has these properties:

Property A. The first partial derivatives of $R$ are squares of rational functions; in fact, for each $m$ from 1 to $n$

$$
\partial R / \partial r_{m}=i_{m}^{2},
$$

where $i_{m}$ is the current flowing in $w_{m}$ when unit current enters the network.

Property B. Let $\alpha_{1}, \alpha_{2}, \cdots, \alpha_{n}$ be any complex numbers. Let $P$ be a permutation of $1,2, \cdots, n$, and denote $P(j)$ by $i_{j}$. Let $r_{i j}$ approach $\alpha_{i_{j}}$ for the successive values $j=1, \cdots, n$. Then $R$ approaches a limit ${ }^{2}$ which is independent of $P$.

Received by the editors March 4, 1949.

${ }^{1}$ For our purposes we do not consider the power source, or the branches joining it to the terminals, to be part of the network. We assume that the network is passive and contains no transformers, though the latter restriction could be dispensed with. We speak of the resistances of the branches, but since we allow these quantities to assume all complex values, the branches may contain impedances.

2 The point at infinity of the complex plane may be the limit of $R$ or may be assigned as the value of an $\alpha_{i}$. We shall describe in detail the process of obtaining the limit, including the conventions which apply to the point at infinity.

Let us substitute successively the $\alpha_{i_{j}}$ for the $r_{i_{j}}$ in numerator and denominator. We assume first that none is infinite. If the denominator does not vanish af ter all substitutions are made, the resulting constant value is evidently the limit of $R$. If at some stage in the course of the substitutions the denominator vanishes identically in the remaining $r_{i j}$, while the numerator becomes a nonzero function of them, we agree to call infinity the limit of $R$. Let us suppose both numerator and denominator become identically zero after the $k$ th substitution, while neither was zero before. Then af ter $k-1$ substitutions $R=U\left(A^{\prime}+B^{\prime} r_{i_{k}}\right) / V\left(M^{\prime}+N^{\prime} r_{i_{k}}\right)$, where $A^{\prime}, B^{\prime}, M^{\prime}, N^{\prime}$ are constants, $B^{\prime} N^{\prime} \neq 0$, and $U$ and $V$ are free of $r_{i_{k}}$. As $r_{i_{k}} \rightarrow \alpha_{i_{k}}$ this function approaches $(U / V) B^{\prime} / N^{\prime}$. We find the limit of $R$ by taking the limit of $U / V$ and multiplying by $B^{\prime} / N^{\prime}$.

Now consider the case that some $\alpha_{i_{k}}$ is infinite, and that a limit is not obtained on account of the vanishing of the denominator only, before the substitution of $\alpha_{i_{k}}$. Then if $r_{i_{k}}$ occurs only in the denominator of the function resulting from the earlier substitutions, the limit of $R$ is zero. If it occurs only in the numerator, we agree to call the limit of $R$ infinity. If it occurs in neither we can disregard it and continue substitutions. Finally, if it occurs in both, we may find the limit of $R$ by omitting from the numerator and denominator the terms free of $r_{i_{k}}$, cancelling $r_{i_{k}}$ from the remaining terms, and continuing the substitutions. 
It may seem unlikely that Property A can be new. ${ }^{3}$ It is intimately related to and easily derived from several well known circuit relations. ${ }^{4}$ Nevertheless equation (1) itself does not appear to be known.

We shall give a proof of Property A which we believe will prove instructive without demanding previous acquaintance with network theory. ${ }^{5}$ We shall derive the necessary preliminary results since this will require but little more space than is needed to state them accurately.

2. Preliminary results. We begin by writing the equations of Kirchhoff for the network $N$ with unit current entering at the terminals. $i_{k}$ denotes, as in the statement of Property A, the current which then flows in $w_{k}$. Let the network contain $s$ nodes or vertices, and $t$ non-self-intersecting loops. We designate the nodes by $v_{1}, v_{2}, \cdots, v_{s}$, where, in particular, $v_{1}$ is the node at which current enters the network, and $v_{s}$ the node at which it leaves. We designate the loops by $l_{1}, l_{2}, \cdots, l_{t}$. We assign a positive direction arbitrarily in each branch $w_{i}$ and each loop $l_{i}$. Then the Kirchhoff equations may be stated as follows:

$$
\begin{array}{ll}
\sum_{k=1}^{n} \epsilon_{j k} i_{k}=\alpha_{j}, & j=1,2, \cdots, s, \\
\sum_{k=1}^{n} \eta_{j k} i_{k} r_{k}=0, & j=1,2, \cdots, t,
\end{array}
$$

where the $\alpha_{j}, \epsilon_{j k}$, and $\eta_{j k}$ have the following values. $\alpha_{1}=1, \alpha_{s}=-1$, $\alpha_{j}=0, j \neq 1, s . \epsilon_{j k}$ is +1 if the $k$ th branch terminates at the $j$ th node, and its positive direction leads to that node, -1 if the $k$ th branch terminates at the $j$ th node, and its positive direction leads from that node, 0 if the $k$ th branch does not terminate at the $j$ th node. $\eta_{j k}=+1$

${ }^{3}$ Since this was written I have seen a proof of a result almost identical with Property A in Montgomery, Dicke, and Purcell, Principles of microwave circuits, McGraw-Hill, 1948, p. 98. It is derived by means of the reciprocity theorem, and used to prove Foster's theorem. There has also appeared a preliminary report of a paper by R. Bott and R. J. Duffin containing, among other results, a proof that Property A holds in a more general situation, and a proof of a partial converse, Bull. Amer. Math. Soc. Abstract 55-3-173.

4 See, for example, Guillemin, Communication networks, Wiley, 1935, vol. 2, pp. 137-139, especially equation 297 on page 139 . My attention was called to this equation by $\mathrm{Mr}$. H. W. Becker, with whom I have had a valuable correspondence concerning the problems discussed in this paper.

${ }^{5}$ In the following discussion I have been guided by a paper of $\mathrm{H}$. Weyl, Reparticion de corriente en una red eléctrica, Revista Matemática Hispano-Americana vol. 5 (1923) pp. 153-164. Our proofs of equations (4) and (6) below are specializations of proofs given there. 
if the $k$ th branch is in the $j$ th loop, and their positive directions coincide, -1 if the $k$ th branch is in the $j$ th loop, and their positive directions are opposed, 0 if the $k$ th branch is not in the $j$ th loop.

It has been shown that the system of equations (2) and (3), which need not be independent, possesses a unique solution for the $i_{k}$ as functions of the $r_{k}{ }^{6}$ If we wish to consider the network as a purely mathematical entity, they may be taken as defining equations for the currents. ${ }^{7}$ It is evident that a change of assignment of positive direction in a branch changes the sign of the current in that branch, and affects no other current.

We define $e_{k}=r_{k} i_{k}$ to be the voltage across a branch $w_{k}$ in the positive direction. The voltage of a vertex $v_{i}$ with respect to a vertex $v_{j}$ is defined as $E_{i j}=\sum \gamma_{k} e_{k}$, where the sum is taken along a path running from $v_{j}$ to $v_{i}$, and $\gamma_{k}$ is +1 or -1 according to whether $w_{k}$ is traversed in the positive or negative direction while describing the path. The value of $E_{i j}$ is independent of the path chosen because of equations (3).

We agree to write $E_{i}$ for $E_{i s}$. Thus $E_{\mathrm{s}}=0$. The resistence $R$ is now defined to be $E_{1} . R$ is independent of the assignment of positive directions in the branches.

We shall now prove the equation

$$
R=\sum_{k=1}^{n} i_{k}^{2} r_{k}
$$

This is the well known equation which states the physically obvious fact that the power consumed by $N$ is the sum of the powers consumed by its branches.

We let $v_{h_{1}}, v_{k_{2}}$ be the vertices of $w_{k}$, and, in particular, choose the notation so that $w_{k}$ is described positively in going from $v_{k_{1}}$ to $v_{k_{2}}$. Then $i_{k}^{2} r_{k}=e_{k} i_{k}=\left(E_{k_{2}}-E_{k_{1}}\right) i_{k}$, so that

$$
\sum_{k=1}^{n} i_{k}^{2} r_{k}=\sum_{k=1}^{n}\left(E_{k_{2}}-E_{k_{1}}\right) i_{k}=\sum_{j=1}^{8}\left(\sum_{k=1}^{n} \epsilon_{j k} i_{k}\right) E_{j} .
$$

The double sum is obtained by grouping terms in the preceding

- More precisely, this means that there exists a unique solution if we regard the $r_{k}$ as indeterminates in the sense of abstract algebra. A proof of this result is contained in the discussion given by F. J. Murray, Theory of mathematical machines, King's Crown Press, New York, 1947, pp. 19-20. In the paper referred to above, Weyl proves that there exists a unique solution for the $i_{k}$ whenever the $r_{k}$ are all positive, from which also our statement can easily be deduced.

' It is evident that a network can be defined, and equations (2) and (3) formulated, using only the language of combinatorial topology. For our purposes it can lead to no difficulty to use the more familiar physical picture and avoid abstract terms. We shall assume, as we obviously may without loss of generality, that our networks are connected, and that each branch terminates at two distinct vertices. 
sum containing the same $E_{j}$; and it is easily seen that the coefficients $\epsilon_{j k}$ answer the description given after (3). Thus the coefficients of $E_{j}$ are zero except for $j=1$ or $s$. Since also $E_{s}=0$, we have $\sum_{k=1}^{n} i_{k}^{2} r_{k}=E_{1}$ $=R$. This is (4).

Let the functions $h_{k}$ of the $r_{i}$ have the property that $\sum_{k=1}^{n} \epsilon_{j k} h_{k}$ $=0, j=1,2, \cdots, s, \epsilon_{j k}$ as in (2). We shall show that

$$
\sum_{k=1}^{n} r_{k} i_{k} h_{k}=0 \text {. }
$$

There is no physically obvious interpretation of (6) as there is of the similar equation (5). However, it may be shown that (6) is equivalent to the minimum dissipation theorem of Maxwell. ${ }^{8}$ To prove (6) we proceed as in the proof of (5). We have $r_{k} i_{k} h_{k}=e_{k} h_{k}=\left(E_{k_{2}}-E_{k_{1}}\right) h_{k}$. Then $\sum_{k=1}^{n} r_{k} i_{k} h_{k}=\sum_{k=1}^{n}\left(E_{k_{2}}-E_{k_{1}}\right) h_{k}=\sum_{j=1}^{s}\left(\sum_{k=1}^{n} \epsilon_{j k} h_{k}\right) E_{j}=0$, on account of the defining properties of the functions $h_{k}$. Thus (6) is proved.

3. Proof of Property A. Having obtained these preliminary results, the proof that $R$ has Property $\mathrm{A}$ is almost immediate. Let $m$ be a fixed integer, $1 \leqq m \leqq n$. On differentiating (2) with respect to $r_{m}$, we see that the functions $\partial i_{k} / \partial r_{m}, k=1,2, \cdots, n$, have the property required of the $h_{k}$. Thus it follows that

$$
\sum_{k=1}^{n} r_{k} i_{k} \partial i_{k} / \partial r_{m}=0 .
$$

If we differentiate (4) with respect to $r_{m}$ we obtain, using (7),

$$
\partial R / \partial r_{m}=2 \sum_{k=1}^{n} r_{k} i_{k} \partial i_{k} / \partial r_{m}+i_{m}^{2}=i_{m}^{2},
$$

and this is (1). This completes the proof.

4. Discussion of Property B. We turn now to property B. We may note that this property appears to have an obvious intuitive interpretation. For, let the $r_{i}$ approach the $\alpha_{i}$ in any order. The resistance $R$ may be expected to vary continuously with the $r_{i}$ so that its value for the $\alpha_{i}$ should be obtained in the limit. But the same network results, and therefore the same $R$ should be obtained, regardless of the order of approach of the $r_{i}$ to the $\alpha_{i}$. Now this is precisely what Property $B$ asserts. Nevertheless the functions $i_{k}$, as is easily seen, do not possess Property $\mathrm{B}$, and even $R$ does not have a unique limit when the $r_{i}$ are allowed to approach the $\alpha_{i}$ along any path. ${ }^{9}$

8 Maxwell, Clerk, $A$ treatise on electricity and magnetism, Clarendon Press, 1873, p. 337.

- In fact, if $R$ possessed a unique limit under all circumstances it would be analytic at all points, and the $i_{k}$, as the square roots of its derivatives, would also 
The function $R$ is known to be linear-fractional. We shall prove Property B by showing that it is a consequence of Property A for linear-fractional functions. In fact we shall prove the following result on the equivalence of these properties:

TheOREM. Let $R$ be a function of $r_{1}, \cdots, r_{n}$ which is linear-fractional in each $r_{i}$. Then $R$ possesses Property $\mathrm{A}$ if and only if it possesses Property B.

5. Proof that A implies B. We shall first prove that Property A implies Property B. Indeed we shall show that if $n-1$ first partial derivatives of the linear-fractional function $R$ are squares of rational functions, then $R$ possesses property B.

We shall use an inductive method of proof. A function of one variable necessarily has property $\mathrm{B}$. We assume therefore that it has been demonstrated that functions of $n-1$ variables, linear-fractional in each, which have $n-2$ derivatives that are squares of rational functions, have Property B. On this basis we proceed to prove our statement concerning $R$. We need consider only the case that $R$ effectively involves $n$ variables.

We may assume that the $\alpha_{i}$ we are dealing with are all zero. For otherwise the substitution $r_{i}^{\prime}=r_{i}-\alpha_{i}$, if $\alpha_{i}$ is finite, $r_{i}^{\prime}=1 / r_{i}$, if $\alpha_{i}$ is the point at infinity of the complex plane, transforms $R$ into a function $R^{\prime}$, linear-fractional in each $r_{i}^{\prime}$, whose first partial derivatives with respect to the $r_{i}^{\prime}$ are squares of rational functions. The limit of $R^{\prime}$ as the $r_{i}^{\prime}$ approach zero in any order equals the limit of $R$ as the $r_{i}$ approach the $\alpha_{i}$ in the same order.

Let us consider the limits approached by $R$ when a particular $r_{i}$, which we shall denote by $r$, is allowed to approach zero first, and the remaining $r_{i}$ then approach zero successively in any order.

We write $R=(A+B r) /(M+N r)$, where $A, B, M$, and $N$ are free of $r$. Evidently $A$ and $M$ cannot both be identically zero, for then $R$ would be independent of $r$. If $M$ is identically zero, $R$ approaches infinity under the circumstances considered. If $M$ is not identically zero, $A / M$ is a linear-fractional function of $n-1$ variables of which at least $n-2$ first partial derivatives are squares of rational functions. By the inductive hypothesis $A / M$ approaches a unique limit when its variables approach zero in any order. But the limits obtained for $R$, after $r$ is set equal to zero, are the limits of $A / M$. Thus, in any case, $R$ approaches a unique limit when $r$ is the first variable to

possess unique limits. A simple example of the phenomenon under consideration is afforded by the resistance $r_{1} r_{2} /\left(r_{1}+r_{2}\right)$ of two wires in parallel. As $r_{1}$ and $r_{2}$ approach zero, this function approaches zero, unless $r_{1}$ and $r_{2}$ vary along a curve tangent to the line $r_{1}+r_{2}=0$. In this exceptional case one may obtain any desired limit by choosing an appropriate curve. 
approach zero, and the remaining variables approach zero in any order.

Let $r, s$ be any two distinct $r_{i}$. To complete the proof it will evidently suffice to consider the limit approached by $R$ as its variables approach zero in the orders $r, s, \cdots$, and $s, r, \cdots$, the ordering of the remaining $r_{i}$ being immaterial.

We write $R=(A+B r+C s+D r s) /(M+N r+P s+Q r s), A, B, C, D$, $M, N, P$, and $Q$ free of $r$ and $s$. If $A$ and $M$ are not both zero, we see as before that $R$ approaches a unique limit in either of the indicated cases. If $A$ and $M$ are both zero, we find by computing $\partial R / \partial s$ that $(N C-B P) r+(N D-B Q) r^{2}$ is the square of a polynomial. This implies that $N C=B P$. Now $B$ and $N$ are not both zero. For, if they were, $s$ would not appear effectively in $R$. Then if $N$ vanishes identically, $P$ vanishes. Similarly if $P$ vanishes, $N$ vanishes. We see then that either $P$ and $N$ are both identically zero, while $B$ and $C$ are not, or $B / N=C / P$.

In the former case $R$ evidently approaches as limit the point at infinity of the complex plane, no matter in which order $r$ and $s$ approach zero. In the latter case the limit of $R$ is the limit of $B / N$, if $s$ approaches zero first, and it is the limit of $C / P$ if $r$ approaches zero first. But these two limits are equal. Thus the limit of $R$ is independent of the order of approach of the variables to zero in all cases. The first implication holds.

6. Proof that B implies A. We shall use the following lemma to prove that Property A is a consequence of Property B:

Let $P$ be a polynomial in variables $x_{1}, \cdots, x_{n}$, quadratic in each of them. If the vanishing of $P$ implies the vanishing of each $\partial P / \partial x_{k}$, $k=1, \cdots, n$, then $P$ is the square of a polynomial.

Since the lemma is easily verified for polynomials in one variable, we may proceed inductively, assuming the truth of the lemma for polynomials in $n-1$ variables, and proving it for $P$.

$P$ is a polynomial which vanishes only when all the derivatives $\partial P / \partial x_{k}, k=1,2, \cdots, n$, vanish. We must prove that it is the square of a polynomial.

If $P=Q R$, where $Q$ and $R$ are each polynomials in one or more variables, and have no variables in common, we see that $Q$ and $R$ each satisfy the condition of the lemma. Then by the inductive hypothesis both $Q$ and $R$ are squares of polynomials. We may thus assume that $\boldsymbol{P}$ has no factorization of this sort. Evidently we may also assume that $P$ involves each $x_{i}$ effectively.

We write $P=A+B x_{n}+C x_{n}^{2}, A, B$, and $C$ free of $x_{n}$. It is easy to see that $C \neq 0$. From the fact that $\partial P / \partial x_{n}$ vanishes whenever $P$ does, we deduce that $B^{2}=4 A C$. Let $T$ be the greatest common divisor of 
$A$ and $C$. We put $A=A_{1} T, C=C_{1} T$. Then $B^{2}=4 A_{1} C_{1} T^{2}$. Since $A_{1}$ and $C_{1}$ have no factors in common each must be the square of a polynomial. Let $A_{1}=U^{2}, C_{1}=V^{2}$. We have $B=2 U V T, P=\left(U+V x_{n}\right)^{2} T$.

No variable occurring in $T$ can occur in $U+V x_{n}$, for then it would appear to at least the third power in $P$. Because of the limitation assumed on the factorization of $P$ we see that either $T$ or $U+V x_{n}$ is a constant. But $U+V x_{n}$ cannot be a constant, for then $P$ would be free of $x_{n}$. It follows that $T$ is a constant, so that $P$ is the square of a polynomial. Thus the lemma is proved.

We now return to the proof of the theorem. We introduce the notation $R=A / D$, where $A$ and $D$ are polynomials in the $r_{i}$ without common factors. We use subscript $k$ to denote partial differentiation of $A$ or $D$ with respect to $r_{k}$. We also write $A^{(k)}, D^{(k)}$ for $A-A_{k} r_{k}$, $D-D_{k} r_{k}$, respectively. These polynomials are evidently independent of $r_{k}$.

We assume that $R$ fulfills the requirement B. We must prove that the partial derivatives of $R$ are perfect squares. This will be accomplished if we can show that the polynomials $A D_{k}-D A_{k}, k=1, \cdots$, $n$, are perfect squares. Now it is easily verified that $A D_{k}-D A_{k}$ is a polynomial quadratic in the $r_{i}$ and free of $r_{k}$. In fact we see that

$$
A D_{k}-D A_{k}=A^{(k)} D_{k}-D^{(k)} A_{k}, \quad k=1, \cdots, n .
$$

We choose some fixed $k$. Consider some set of values of the $r_{i}$ other than $r_{k}$, say, $r_{i}=s_{i}, i \neq k$, which annul $A D_{k}-D A_{k}$. We know from the lemma that it will suffice to show that each derivative $\partial\left(A D_{k}-D A_{k}\right) / \partial r_{j}, j \neq k$, vanishes for these values. For this purpose we assign a value $s_{k}$ to $r_{k}$ as follows:

(a) If neither $A_{k}$ nor $D_{k}$ is annulled by the $s_{i}$, $i \neq k$, we let $s_{k}=-A^{(k)} / A_{k}$, noting that $A^{(k)} / A_{k}=D^{(k)} / D_{k}$, when the $s_{i}$ are substituted for the $r_{i}$.

(b) If $D_{k}$ vanishes when the $r_{i}, i \neq k$, are replaced by the $s_{i}$, but $A_{k}$ does not, we let $s_{k}=-A^{(k)} / A_{k}$; and note that $D^{(k)}$ must also vanish.

(c) If $A_{k}$ vanishes when the $r_{i}, i \neq k$, are replaced by the $s_{i}$, but $D_{k}$ does not, we let $s_{k}=-D^{(k)} / D_{k}$, and note that $A^{(k)}$ must also vanish.

(d) If both $A_{k}$ and $D_{k}$ vanish when the $r_{i}, i \neq k$, are replaced by the $s_{i}$, we assign no value to $r_{k}$.

We observe that in the first three cases both $A$ and $D$ vanish when the $r_{i}$ are replaced by the $s_{i}, i=1, \cdots, n$.

We shall now show that $A_{k} D_{j}-D_{k} A_{j}$, where $j$ is a positive integer not exceeding $n$ and distinct from $k$, vanishes when the $r_{i}$ are replaced by the $s_{i}$. In case (a), $A$ and $D$ vanish. The unique limit of $A / D$ is given by $A_{k} / D_{k}$, and also by the limit of $A_{j} / D_{j}$. If $D_{j}$ does not vanish, we must have $A_{k} / D_{k}=A_{j} / D_{j}$, after the substitution of the $s_{i}$. If $D_{j}$ vanishes, then $A_{j}$ must vanish too, in order that the 
limit of $A_{j} / D_{j}$ may equal the finite value of $A_{k} / D_{k}$. Under either circumstance our statement concerning $A_{k} D_{j}-D_{k} A_{j}$ is obviously true. In case (b), $A$ and $D$ again vanish, and this again implies that $A_{k} / D_{k}$ and $A_{j} / D_{j}$ both have the same limit. But this means that $D_{j}$ as well as $D_{k}$ must be annulled by the $s_{i}$. Similarly, in case (c) both $A_{j}$ and $A_{k}$ vanish. Thus in either of these cases our statement is true. In case (d) it is obvious.

With these observations we can now complete the proof that $\partial\left(A D_{k}-D A_{k}\right) / \partial r_{j}$ is annulled by the $s_{i}$. We have,

$$
\frac{\partial}{\partial r_{j}}\left(A D_{k}-D A_{k}\right)=\left(A D_{k j}-D A_{k j}\right)+\left(A_{j} D_{k}-D_{k} A_{j}\right) .
$$

We have just shown that the term in the second bracket on the right is annulled by the $s_{i}$. In cases (a), (b), and (c) the first bracket is known to vanish since $A$ and $D$ are annulled. It remains only to prove that the first bracket vanishes also in case (d).

We may assume that the polynomials $A, D$ are not both zero, and also that $A_{k j}, D_{k j}$ are not both zero after the $r_{i}$ are replaced by the $s_{i}$. We write

$$
A / D=\left(E+F r_{j}+G r_{k}+H r_{j} r_{k}\right) /\left(M+N r_{j}+P r_{k}+Q r_{j} r_{k}\right),
$$

$E, F, G, H, M, N, P, Q$ free of $r_{j}$ and $r_{k}$. Evidently $H=A_{k j}, Q=D_{k j}$. We see that $G+H r_{j}=P+Q r_{j}=0$, when the $r_{i}$ are replaced by the $s_{i}$. It follows that $E+F r_{j}, M+N r_{j}$ are not both zero after these replacements. Finally $H$ and $Q$ are not both zero after the substitutions.

We consider the limit of $R$ as $r_{k}$ approaches infinity and the remaining $r_{i}$ approach $s_{i}$. If $r_{k}$ approaches infinity before the other variables approach their limits, we obtain $\lim R=\lim \left(G+H r_{j}\right) /\left(P+Q r_{j}\right)$, and this is the value assumed by $H / Q$ when the $r_{i}$ are replaced by $s_{i}$, or infinity if $Q=0 .{ }^{10}$ If we let the $r_{i}$ approach $s_{i}$ before $r_{k}$ approaches infinity, we have that $R$ approaches the value taken by $\left(E+F r_{j}\right) /\left(M+N r_{j}\right)$ when the $r_{i}$ are replaced by $s_{i}$, or that $R$ approaches infinity if $M+N r_{j}$ becomes zero after these substitutions. The equality of the limits now shows that either $\left(E+F r_{j}\right) /\left(M+N r_{j}\right)$ $=H / Q$, or $M+N r_{j}=Q=0$ after the replacements. In either case we have $\left(E+F r_{j}\right) Q=\left(M+N r_{j}\right) H$, and therefore $\left(E+F r_{j}+G r_{k}+H r_{j} r_{k}\right) Q$ $-\left(M+N r_{j}+P r_{k}+Q r_{j} r_{k}\right) H=0$ after the replacements. But the lefthand side of this expression is precisely $A D_{k j}-D A_{k j}$.

${ }^{10}$ For the limit may be computed by substituting the $s_{i}$ for the $r_{i}$ in any order. Let the substitution of $s_{j}$ for $r_{j}$ be made last. If $Q$ vanishes, then so does $P$, but not $H$, and we obtain, at the step before replacement of $r_{j}$, the value infinity for the limit. If $Q$ does not vanish, L'Hôpital's rule, which coincides with the procedure of footnote 2 , gives the stated value. 
We have thus seen that in all cases $\partial\left(A D_{k}-D A_{k}\right) / \partial r_{j}$ is annulled by the $s_{i}$. The lemma now shows that $A D_{k}-D A_{k}$ is a perfect square. This completes the proof that Property B implies Property A.

Corollary. If a function $R$, linear-fractional in its $n$ variables $r_{1}, r_{2}, \cdots, r_{n}$, has $n-1$ first partial derivatives which are squares of rational functions, then it possesses Property B, and all its first partial derivatives are squares of rational functions.

Since Property B is implied by the fact that $n-1$ first partial derivatives are perfect squares, and since it in turn implies Property A, the corollary follows immediately.

7. Conclusion. It was our original hope that Property A, or its equivalent $B$, together with the obvious requirements

(a) that $R$ be linear homogeneous,

(b) that it be linear-fractional in each $r_{i}$,

(c) that the coefficients of each term in the numerator and denominator be +1 after all common factors have been removed,

would be necessary and sufficient conditions for $R$ to be the resistance of a two-terminal network composed of the $n$ branches $w_{1}$, $w_{2}, \cdots, w_{n}$, having the independently variable resistances $r_{1}, r_{2}, \cdots$, $r_{n}$ respectively. However these conditions are not sufficient as the following considerations show. ${ }^{11}$

Let $R$ satisfy the conditions stated above. Let $s_{i}=1 / r_{i}, S=1 / R$. Then $S$, as a function of the $s_{i}$, satisfies these conditions, and is termed the dual of $R$. To any planar ${ }^{12}$ network $N$ there exists a conjugate network $N^{\prime}$ which contains a vertex corresponding to each loop, and a loop corresponding to each vertex of $N$. The resistance of $N^{\prime}$ is the dual of the resistance of $N$. Nonplanar networks possess no conjugates, so that one is led to surmise that the duals of their resistances are not the resistance of any network. We may construct a nonplanar network by joining six branches in the form of a hexagon and connecting two additional branches between pairs of opposite vertices. Current enters and leaves at the remaining vertices. Our surmise is easily verified for the dual of the resistance of this network. Thus this dual is a function meeting the stated conditions, yet not the resistance of any network.

RUTGERS UNIVERSITY

"A converse result of this type has been obtained, however, in a more general situation by R. Bott and R. J. Duffin; see footnote 3. We are indebted to Mr. Becker for the counterexample given below.

12 A network, in the sense defined in (1), is planar if it can be represented in a plane, together with a branch joining its terminals, in such a manner that no branches meet except at their common vertices. 